\title{
More Is Not Always Better: Increased Fractional Anisotropy of Superior Longitudinal Fasciculus Associated with Poor Visuospatial Abilities in Williams Syndrome
}

\author{
Fumiko Hoeft, ${ }^{1}$ Naama Barnea-Goraly, ${ }^{1}$ Brian W. Haas, ${ }^{1}$ Golijeh Golarai, ${ }^{1}$ Derek Ng, ${ }^{1}$ Debra Mills, ${ }^{2}$ Julie Korenberg,, 3 \\ Ursula Bellugi, ${ }^{5}$ Albert Galaburda, ${ }^{6}$ and Allan L. Reiss ${ }^{1}$ \\ ${ }^{1}$ Center for Interdisciplinary Brain Sciences Research, Department of Psychiatry and Behavioral Sciences, Stanford University School of Medicine, Palo Alto, \\ California 94305-5795, 2 Department of Psychology, Emory University, Atlanta, Georgia 30322, ${ }^{3}$ Department of Pediatrics, Cedars Sinai Medical Center, Los \\ Angeles, California 90048, ${ }^{4}$ Department of Human Genetics, David Geffen School of Medicine at the University of California, Los Angeles, Los Angeles, \\ California 90095-7088, ${ }^{5}$ Laboratory for Cognitive Neuroscience, Salk Institute for Biological Studies, La Jolla, California 92037, and ${ }^{6}$ Department of \\ Neurology, Beth Israel Deaconess Medical Center, Harvard Medical School, Boston, Massachusetts 02215
}

We used diffusion tensor imaging to examine white matter integrity in the dorsal and ventral streams among individuals with Williams syndrome (WS) compared with two control groups (typically developing and developmentally delayed) and using three separate analysis methods (whole brain, region of interest, and fiber tractography). All analysis methods consistently showed that fractional anisotropy (FA; a measure of microstructural integrity) was higher in the right superior longitudinal fasciculus (SLF) in WS compared with both control groups. There was a significant association with deficits in visuospatial construction and higher FA in WS individuals. Comparable increases in FA across analytic methods were not observed in the left SLF or the bilateral inferior longitudinal fasciculus in WS subjects. Together, these findings suggest a specific role of right SLF abnormality in visuospatial construction deficits in WS.

Key words: Williams syndrome; visuospatial construction; superior longitudinal fasciculus; diffusion tensor imaging; developmental disabilities; genetics

\section{Introduction}

Williams syndrome (WS), a neurodevelopmental disorder caused by a hemizygous deletion of up to 28 genes on chromosome 7q11.23 (Hillier et al., 2003), offers a unique opportunity to investigate interplays between gene, brain, and behavior. Cognitive hallmarks of WS include severe visuospatial deficits and relative strengths in face and object processing (Meyer-Lindenberg et al., 2006). Functional neuroimaging data has indicated anomalous functional connectivity of the dorsal stream in WS, suggesting that aberrant connectivity of dorsal stream white matter tracts may be one link between genetic and visuospatial abnormalities in WS. However, previous studies have not directly addressed this possibility.

In this study we used diffusion tensor imaging (DTI) to examine white matter integrity of two major white matter pathways, namely the superior longitudinal fasciculus (SLF), which is associated with the dorsal stream, i.e., the "where" pathway, and the

\section{Received Aug. 8, 2007; revised Sept. 11, 2007; accepted Sept. 11, 2007}

This work was supported by National Institute of Child Health and Human Development Grant P01 HD033113-12. We thank Dr. Susumu Mori for comments on a previous version of this manuscript and Drs. Susumu Mori (DtiStudio), Bruce McCandliss, and Sumit Niogi (ROQS) for providing their software.

Correspondence should be addressed to Fumiko Hoeft, Center for Interdisciplinary Brain Sciences Research, Department of Psychiatry and Behavioral Sciences, Stanford University School of Medicine, 401 Quarry Road, Palo Alto, CA 94305-5795. E-mail: fumiko@stanford.edu.

DOI:10.1523/JNEUROSCI.3591-07.2007

Copyright $\odot 2007$ Society for Neuroscience $\quad$ 0270-6474/07/2711960-06\$15.00/0 inferior longitudinal fasciculus (ILF), which is associated with the ventral stream, i.e., the "what" pathway. We hypothesized that WS is associated with abnormal white matter integrity along the SLF, especially in the right hemisphere, given the right lateralization of visuospatial functions along the dorsal stream and deficits in these functions among persons with WS (Makris et al., 2005; Tuch et al., 2005). In contrast, we expected the ILF to be less affected in WS, given its putative role in face and object processing and the relative strength in these processes observed in WS. Furthermore, we hypothesized that the degree of SLF anomalies in WS would be specifically correlated to level of dysfunction in visuospatial construction among individuals with WS.

\section{Materials and Methods}

\section{Participants}

A total of 20 WS, 11 typically developing (TD), and 10 developmentally delayed (DD) individuals participated in the study. WS subjects were recruited as part of an ongoing program grant (HD33113), which includes event-related-potential, behavioral, molecular genetics, histologic, and volumetric magnetic resonance imaging (MRI) studies (Reiss et al., 2000; Galaburda et al., 2001; Schmitt et al., 2001a,b,c, 2002; Levitin et al., 2003; Mobbs et al., 2004, 2006; Eckert et al., 2005, 2006a,b,c; Holinger et al., 2005; Thompson et al., 2005; Gaser et al., 2006) (for overview, see Bellugi and St. George, 2000). All genetic diagnoses were confirmed using florescent in situ hybridization probes for ELN (elastin), a gene consistently found in the microdeletion associated with WS (Ewart et al., 1993; Korenberg et al., 2003). In addition, all participants exhibited the medical and clinical features of the WS phenotype, includ- 
Table 1. Demographic information

\begin{tabular}{|c|c|c|c|c|c|}
\hline & & \multicolumn{2}{|l|}{ WS } & \multicolumn{2}{|c|}{ Controls } \\
\hline & & All & Matched & DD & TD \\
\hline \multicolumn{2}{|l|}{$n$} & 20 & 10 & 10 & 10 \\
\hline \multirow{3}{*}{$\begin{array}{l}\text { Handedness } \\
\text { Gender }{ }^{*}\end{array}$} & Left:right & $2: 18$ & $1: 9$ & $1: 9$ & $0: 10$ \\
\hline & Female:male & $8: 12$ & $4: 6$ & $7: 3$ & $3: 7$ \\
\hline & Mean & 31.8 & 26.8 & 23.2 & 27.8 \\
\hline \multirow[t]{2}{*}{ Age } & SD & 10.8 & 7.5 & 5.5 & 9.5 \\
\hline & Mean & 69.9 & 71.7 & 69.5 & 114.3 \\
\hline \multirow[t]{2}{*}{ Verbal IQ* } & SD & 11.0 & 9.9 & 13.9 & 12.1 \\
\hline & Mean & 64.7 & 65.7 & 77.1 & 110.6 \\
\hline \multirow[t]{2}{*}{ Performance $1 Q^{*}$} & SD & 9.3 & 8.2 & 18.8 & 14.0 \\
\hline & Mean & 65.4 & 67.2 & 71.1 & 114.3 \\
\hline Full-Scale IQ* & SD & 10.6 & 8.9 & 16.2 & 12.8 \\
\hline
\end{tabular}

$\sim{ }^{*} p<0.1 ;{ }^{*} p<0.001$; IQ is missing from one TD subject.

ing cognitive, behavioral, and physical profiles (Committee on Genetics, American Academy of Pediatrics, 2001; Meyer-Lindenberg et al., 2006).

TD subjects were screened for a history of psychiatric or neurologic problems using the Symptom Checklist-90-R (SCL-90-R) (Derogatis, 1977). All subjects had SCL-90-R scores that fell within one SD of a normative sample. Cognitive functioning was assessed by using the Wechsler Adult Intelligence Scale, third edition (WAIS-III). It assessed verbal intelligence quotient (VIQ), performance intelligence quotient (PIQ), and full-scale intelligence quotient (FSIQ).

Criteria for the DD control group were met if participants' full-scale intelligence quotient (IQ) fell below one SD of the norm and participants did not have the diagnosis of WS. Among the DD individuals, seven had idiopathic DD, and three had a diagnosis of fragile X syndrome, Turner syndrome, or velocardiofacial syndrome, and were diagnosed as in previous publications from our group (Menon et al., 2004; Gothelf et al., 2005, 2007a,b; Thompson et al., 2005; Holzapfel et al., 2006; Kesler et al., 2006; Hoeft et al., 2007).

Among these individuals, 10 individuals from each group were then selected based purely on their demographic information (age, gender, and handedness) without any information about their DTI data to obtain three demographically matched groups. DTI data of these groups were compared using three different DTI analysis techniques to examine between-group differences in white matter integrity. In a second set of analysis, data from all WS individuals (20 subjects) were used to examine covariation with white matter integrity and cognitive profiles.

A summary of the participants' demographic information is listed in Table 1. There was a trend for significant difference in the proportion of males to females among the three groups $\left(\chi_{(2)}^{2}=5.16, p=0.08\right)$, which was not driven by the comparisons of interest [WS vs TD $(p=0.33)$, WS vs DD $(p=0.18)]$. There were no significant differences in age $\left(F_{(2,27)}=\right.$ $0.95, p=0.39$ ) or the proportion of left- to right-handed individuals among the three groups $\left(\chi_{(2)}^{2}=3.22, p=0.52\right)$. VIQ, PIQ and FSIQ showed main effects of group $\left(F_{(2,27)}=40.75, p<0.001 ; F_{(2,27)}=24.62\right.$, $p<0.001$; and $F_{(2,27)}=38.74, p<0.001$, respectively). The differences were driven by the significant differences between the WS and TD groups (all $p$ values $<0.001$ ) but not between the WS and DD groups ( $p=0.97$, 0.24 , and 0.88 , respectively).

None of the participants had a contraindication for MRI, and written informed consent and assent were obtained according to the Declaration of Helsinki. This study was approved by the Stanford University Administrative Panel on Human Subjects in Medical Research, and informed consent/assent were obtained.

\section{DTI image acquisition}

Magnetic resonance images of each subject's brain were acquired the Lucas Center (Stanford University, Palo Alto, CA) using a 3T Signa LX (GE Medical Systems, Milwaukee, WI). A DTI sequence was based on a single-shot spin-echo echo-planar imaging sequence with diffusion sensitizing gradients applied on either side of the $180^{\circ}$ refocusing pulse (Basser et al., 1994). Imaging parameters for the diffusion-weighted sequence were as follows: field of view (FOV), $24 \mathrm{~cm}$; matrix size, $128 \times 128$ (33 slices); echo time (TE), $60.4 \mathrm{~ms}$; repetition time (TR), 12,200 ms; 33 axial-oblique slices; slice thickness, $3.8 \mathrm{~mm} / \mathrm{skip} 0.4 \mathrm{~mm}$. Diffusion gradient duration was $\delta=32 \mathrm{~ms}$, and diffusion weighting was $b=815$ $\mathrm{s} / \mathrm{mm}^{2}$. In addition, two reference measurements ( $B 0$ scans) were performed and averaged for each slice after removing the diffusion sensitizing gradients. Diffusion was measured along six noncollinear directions: $X Y, X Z, Y Z,-X Y,-X Z$, and $-Y Z$. This pattern was repeated six times for each slice, with the sign of all diffusion gradients inverted for odd repetitions. To aid in the localization of white matter differences, a threedimensional, high-resolution T1-weighted anatomic gradient and a receptive field-spoiled gradient recall, MRI sequence with the following parameters was used: TR, $35 \mathrm{~ms}$; TE, $6 \mathrm{~ms}$; flip angle, $45^{\circ}$; number of excitations, 1 ; matrix size, $256 \times 256$; FOV, $24 \mathrm{~cm}^{2}$; 124 contiguous slices of $1.5 \mathrm{~mm}$ width.

\section{DTI image processing}

Preprocessing. First diffusion-weighted images were corrected for eddy current distortions and head motion using linear image registration [automated image registration (AIR) algorithm] (Woods et al., 1998) [note that the reproducible objective quantification scheme (ROQS) method below was not corrected for head motion]. Thereafter, DtiStudio (Jiang et al., 2006) (https://www.dtistudio.org/) was used. All individual images were then visually inspected to discard slices with motion artifacts, after which the remaining images were added for each slice. The pixel intensities of the multiple diffusion-weighted images were then fitted to obtain the six elements of the symmetric diffusion tensor. The diffusion tensors at each pixel were diagonalized to obtain pixel eigenvalues and eigenvectors. Fractional anisotropy (FA) maps, average non-diffusion-weighted images $\left(b=0 \mathrm{~s} / \mathrm{mm}^{2}\right)$, average diffusion coefficient images (ADC; $b=$ $\left.815 \mathrm{~s} / \mathrm{mm}^{2}\right)$, and primary eigenvectors [eigenvector $\left(\mathrm{v}_{1}\right)$ associated with the largest eigenvalue $\left(\lambda_{1}\right)$ was assumed to represent the local fiber direction] of the diffusion tensor were obtained for additional analyses.

Voxel-based analysis using tract-based spatial statistics. For voxelwise analysis of FA, tract-based spatial statistics (TBSS) (Smith et al., 2006), which is part of FSL (Smith et al., 2004) (http://www.fmrib.ox.ac.uk/fsl/), was used to perform whole-brain analysis of white matter FA. FA maps from each individual were coregistered using nonlinear registration IRTK (Rueckert et al., 1999) (www.doc.ic.ac.uk/ dr/software) to the subject closest to the group mean. After image registration, FA maps were averaged to produce a group mean FA image. A skeletonization algorithm was applied to the group mean FA image to define a group template of the lines of maximum FA, thought to correspond to centers of white matter tracts. FA values for each individual subject were then projected onto the group template skeleton by searching along perpendiculars from the skeleton to find local maxima. Voxelwise analyses of FA across the group of subjects were performed only on data projected onto the skeleton template (which is recruited from the nearest tract center in each subject's image).

ROQS. To avoid several assumptions and potential confounds associated with realignment and spatial normalization of white matter tracts, this second analysis adopted a region of interest (ROI) approach to test two specific regions within each hemisphere selected in an a priori manner from previous studies reporting associations between FA and visuospatial ability and face processing (Schmahmann and Pandya, 2006). These selected structures included the following: right- and lefthemisphere superior and inferior longitudinal fasciculi (SLF and ILF, respectively).

These ROIs were selected using ROQS, a semiautomated process that segments white matter structures based on user-selected seed pixels (Niogi et al., 2007), by an investigator blind to subjects' diagnoses. ROQS operates in a four-step process using directionally encoded information from the principal eigenvector to segment structures that the user first selects by assigning a seed pixel. The second step is to determine thresholds and selection criteria based on properties of the seed. During this step, the ROQS algorithm determines the $x, y$, and $z$ components of the principal eigenvector of the seed pixel, where the principal eigenvector denotes the direction of maximal diffusivity. ROQS restricts the selection to pixels with the same maximum component $(x, y$, or $z)$ of the principal eigenvector of the seed pixel. The third step is to create a binary mask such that pixels that fit the criteria determined in the second step are 


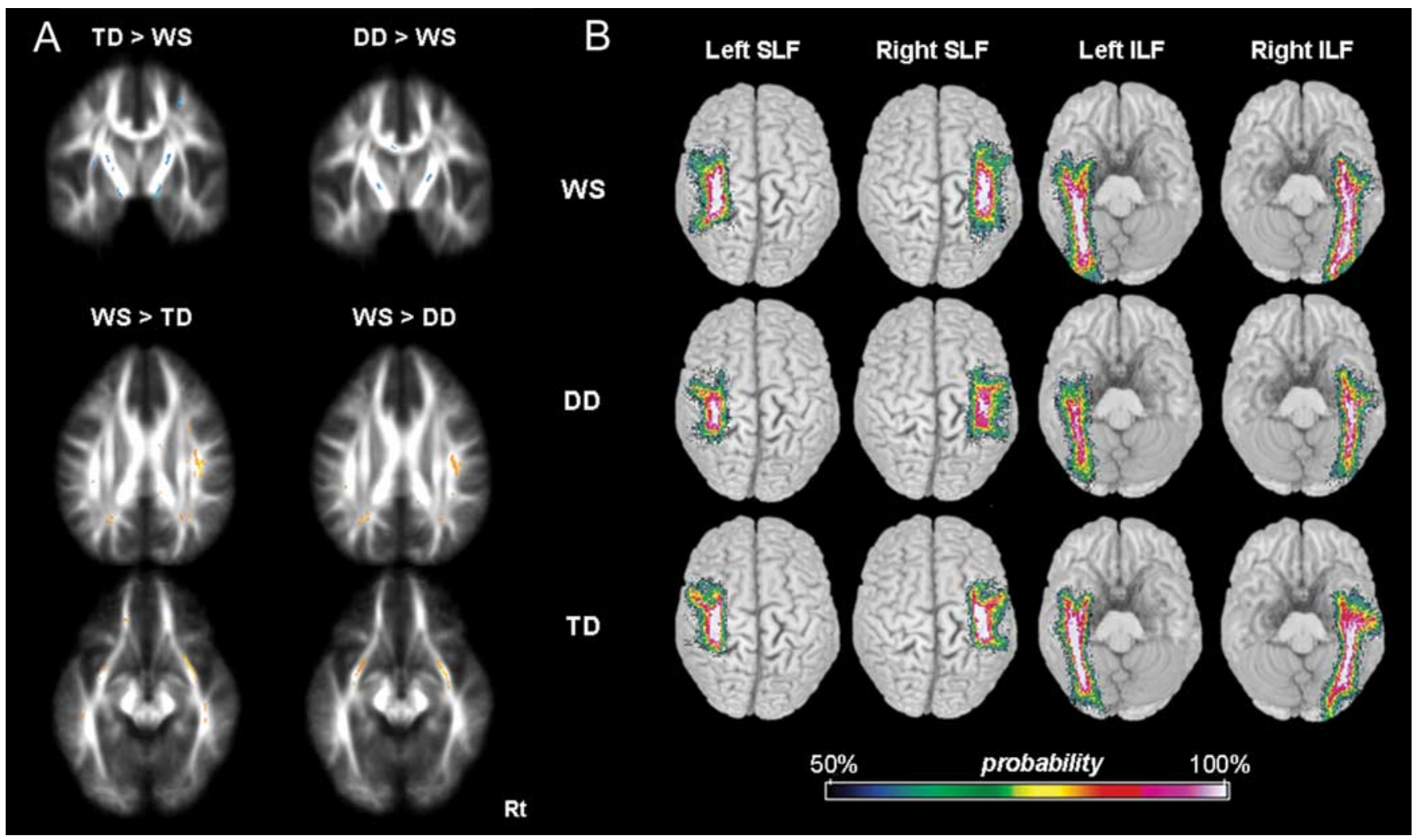

Figure 1. Results overlaid on whole brain. $A$, TBSS voxel-based analysis results overlaid on FA template in coronal (top row) and axial views (second and third rows). Regions that show significant difference in FA values between WS and TD and between WS and DD groups are shown. Top row shows PLIC/CPT/STR/SCT regions (Talairach coordinate, $y=-8)$, middle row shows $S L F$ region $(z=$ $29)$, and bottom row shows UF/IFO regions $(z=-12) . p=0.05$ corrected. $B$, Probabilistic maps of SLF and ILF for each group overlaid on a three-dimensional whole-brain T1 image. The SLF is projected onto the top of the brain, and the ILF is projected onto the bottom of the brain. A value of $100 \%$ indicates that all subjects in the group show overlapping tracts in that particular voxel. Right hemisphere is shown on right ( $\mathrm{Rt})$.

assigned a value of one and all other pixels are assigned a value of zero. The final step is for ROQS to determine the boundary of the structure. This occurs by drawing a vector from the seed pixel to a pixel with a value of zero. A chain algorithm is then applied to determine and connect all boundary pixels. The final ROI includes all pixels within this boundary. The benefit of the ROQS analysis is that regions conforming to the boundaries of the tracts are selected using an objective, reproducible algorithm, in a manner specific to each subject. Selection and analysis was implemented with software written in Interactive Data Language v6.0 (IDL; Research Systems, Boulder, CO). The average and SD of the FA as well as ADC is calculated for each ROI for each run and for each subject.

One feature of ROQS is that each subject contributes a uniquely sized and shaped ROI for a given structure. It also selects ROIs at and near the seed voxel, as opposed to the whole fiber tract or in a voxel-by-voxel manner. To complement these steps, FA measurements of the entire tracts of interest using diffusion-tensor fiber tracking was also performed.

Diffusion-tensor fiber tracking. All analyses were performed using DtiStudio (Mori et al., 2002). Fiber tracking was performed using the fiber assignment by continuous tracking method (Mori et al., 1999). Briefly, tracing was initiated from a seed pixel from which a line was propagated in both retrograde and orthograde directions according to $v_{1}$ at each pixel. The tracking was terminated when it reached a pixel with an FA value lower than 0.15 or when the turning angle was $>40^{\circ}$ (Wakana et al., 2004). To reconstruct branching patterns, the tracking was performed from every pixel inside the brain, but only fibers that penetrated ROIs defined from anatomical landmarks were retained (Conturo et al., 1999).

To identify the SLF and ILF, ROIs were drawn for each subject based on anatomical landmarks described by Mori et al. (2002) and Wakana et al. (2004), by an investigator blind to the subjects' diagnoses. Briefly, the ROI for the SLF was defined by navigating coronally to the posterior tip of the putamen and selecting voxels on the color map identified as the SLF (superior/lateral to corpus callosum and superior to lateral sulcus). Individual fibers were defined as the SLF if they projected through this ROI and did not project into the opposing hemisphere. The ILF was identified by using a two-ROI approach (Mori et al., 2002). One ROI was drawn on a coronal slice identified by the parieto-occipital sulcus to include the entire occipital lobe. The second ROI was drawn on a coronal slice identified by the posterior tip of the putamen to include the entire midtemporal lobe. Individual fibers were defined as the ILF if they projected through both of these ROIs and did not project into the frontal lobe or the opposing hemisphere. One drawback of this anatomy-based, double-ROI reference approach is its inability to reconstruct branching between chosen pairs of ROIs; as a consequence, it is mainly suitable for tracts that connect two distant regions and do not branch out between the two target areas. Therefore, for SLF, which is known to have extensive branching in the frontal, parietal, and temporal lobes, only one ROI was used defined on a color-coded map.

Probabilistic maps of diffusion-tensor fiber tracking. To analyze the reliability of the tracking methods described above in Diffusion-tensor fiber tracking, and to determine normal and pathological variations in patterns of these reconstructed axonal tracts and their association with different cortical areas (e.g., branching), statistical maps for each tract system were generated (Mori et al., 2002). To achieve this, we standardized individual B0 images into the template T2 image that is in the Montreal Neurological Institute coordinate brain reference frame using algorithms implemented in Statistical Parametric Mapping 2 (SPM2; Wellcome Department of Cognitive Neurology, London, UK; http://www.fil.ion.ucl.ac.uk/spm/). We then calculated the probability of having a particular tract system delineated as explained above for each pixel for each group. In these probabilistic maps, each pixel reflects the percentage of normal subjects that contained a particular tract. This approach allows us to visualize the consistency of the core as well as some of the branches. 
Covariation between white matter abnormality and cognitive profiles in WS individuals. To examine domain specificity, we examined correlations between visuospatial ability (WAIS-III Object Assembly subtest) and FA values obtained from ROQS. These cognitive measures were only obtained in WS individuals. For this analysis, we included all 20 WS individuals with usable data to increase power.

Statistical analysis. Statistical analysis of behavioral and demographic data and ROQS and fiber-tracking results were performed using Matlab (Mathworks, Natick, MA). Group statistics were performed using oneway ANOVA and post hoc contrasts, two-sample $t$ tests, and Pearson correlation for parametric data (none of the statistics included nonparametric data; hence, nonparametric statistics were not performed). For TBSS, we tested for significant differences in FA between WS and TD groups and between WS and DD groups using general linear models (two-sample $t$ tests). Correction for multiple comparisons was performed using permutation-based inference (Nichols and Holmes, 2002) with a cluster-forming threshold of $t=3$ and a corrected cluster size significance level of $p<0.05$ voxel-based thresholding corrected for multiple comparisons by using the null distribution of the max (across the image) test statistic.

\section{Results}

First, we used an automated observer-independent approach for assessing groupwise microstructural differences in the major white matter pathways throughout the brain and performed whole-brain voxel-based analysis on normalized DTI images with TBSS (Smith et al., 2006) (Fig. 1A). WS compared to TD showed greater FA in bilateral SLF (right $>$ left), right superior fronto-occipital fasciculus, bilateral external capsule (EC)/uncinate fasciculus (UF)/inferior fronto-occipital fasciculus (IFO), bilateral ILF/IFO, and bilateral forceps major. TD compared with WS showed greater FA in white matter regions within the splenium of the corpus callosum and bilateral posterior limbs of the internal capsule (PLIC)/corticopontine tract (CPT)/corticospinal tract (CST) and superior thalamic radiation (STR).

We then examined differences between WS and DD individuals. Results were very similar to the WS/TD comparison. WS compared to DD showed higher FA in bilateral SLF (right $>$ left), right SFO, bilateral EC/UF/IFO, bilateral ILF/IFO, and bilateral forceps major. In DD compared with WS, FA values were higher in body and splenium of the corpus callosum, and bilateral PLIC/CPT/CST/STR.

The TBSS methods that we used minimize confounds associated with preprocessing (e.g., realignment and smoothing). To further examine more specifically across methods, thus overcoming limitations specific to each method, the role of SLF and ILF in visuospatial deficits among WS, two additional analyses were performed in each subject's native space: (1) ROQS, a semiautomated process that segments white matter structures based on user-selected seed pixels and that has been shown to have higher inter- and intra-rater reliability than manual tracings of anatomical regions (Niogi et al., 2007) and (2) fiber tracking using previously described methods (Wakana et al., 2004).

Using ROQS, we found that FA in right SLF was higher in WS than in controls $\left(F_{(2,27)}=4.69, p=0.018\right.$; WS $>$ TD: $t_{(27)}=2.17$, $p=0.047$; WS $>$ DD: $\left.t_{(27)}=2.66, p=0.016\right)$ (Fig. $2 A$, Table 2). We found corresponding decreases in ADC in right SLF in WS $\left(F_{(2,27)}=6.29, p=0.006\right.$; WS $<$ TD: $t_{(27)}=2.46, p=0.024$; WS $<$ DD: $\left.t_{(27)}=3.39, p=0.003\right)$. Although right ILF also showed higher FA in WS compared with controls $\left(F_{(2,27)}=6.34\right.$, $p=0.006$; WS $>$ TD: $t_{(27)}=3.12, p=0.006$; WS > DD: $t_{(27)}=$ $3.26, p=0.006)$, there were no corresponding between-group differences in $\operatorname{ADC}\left(F_{(2,27)}=0.09, p=0.92\right)$. Left SLF and bilateral ILF showed no significant differences between WS and controls $(p=0.24-0.92)$.

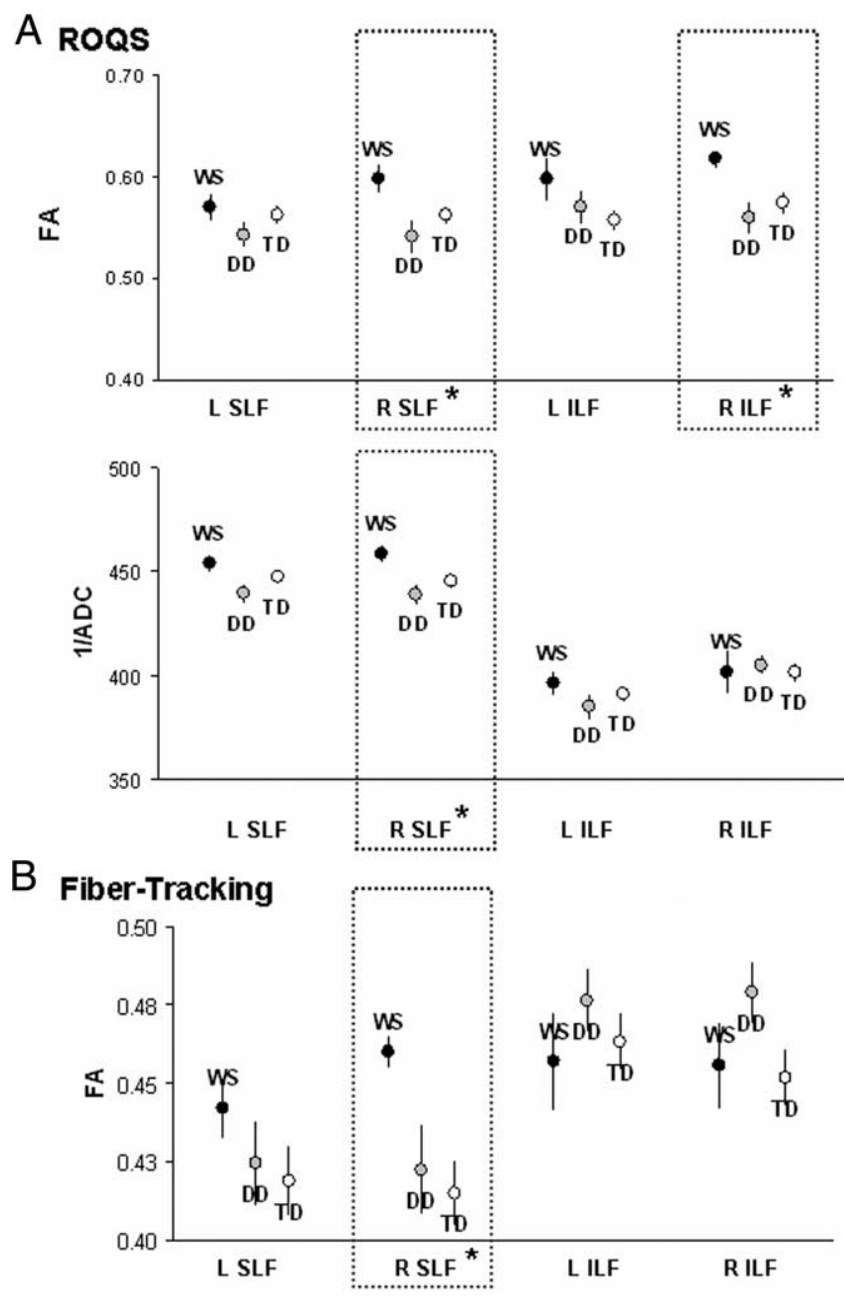

Figure 2. FA and $A D C$ results. Mean FA and $1 / A D C$ values from ROQS $(\boldsymbol{A})$ and mean FA values from fiber tracking $(\boldsymbol{B})$ are plotted for each fiber tract: left superior longitudinal fasciculus ( $L$ SLF), right SLF (R SLF), L inferior longitudinal fasciculus (L ILF), and R ILF. Error bar represent SEMs. * indicates fibers that showed a main effect of group using one-way ANOVA, and significant differences between WS and TD and between WS and DD (Table 2).

Consistent with these findings, fiber-tracking methods revealed higher FA in right SLF among WS than controls $\left(F_{(2,27)}=\right.$ $5.17, p=0.013$; WS $>$ TD: $t_{(27)}=3.90, p=0.002$; WS > DD: $t_{(27)}$ $=2.48, p=0.030$ ), but no between-group differences for other tracts $(p=0.19-0.50)$ (Fig. 2B, Table 2). Probabilistic maps of these tracts are presented in Figure $1 B$.

Although we attempted to match the three groups as much as possible, there were insignificant yet small differences (e.g., female-to-male ratio and handedness) between the TD and DD groups. We hence performed additional analyses by creating two subgroups. One subgroup consisted of 10 WS individuals exactly matched for gender and handedness with the 10 TD individuals, whereas the other was matched to the 10 DD individuals (WS vs TD and WS vs DD: $\left.\chi_{(2)}^{2}=0.00, p=1.00\right)$. Age was not significantly different between these WS subgroups and corresponding TD and DD groups, and IQ groups. When FA of the right SLF was compared between these groups, the results were essentially unchanged from previous analyses, showing greater FA in WS compared with both TD and DD (WS vs TD: ROQS, $t_{(18)}=2.65$, 0.016 , fiber tracking, $t_{(18)}=3.01, p=0.012$; WS vs DD: ROQS, $t_{(18)}=2.49, p=0.025$, fiber tracking, $\left.t_{(18)}=2.34, p=0.036\right)$.

To examine the potential role of white matter abnormalities in 
the cognitive characteristics of WS, we examined the covariance of FA (from ROQS analysis) and measures of visuospatial abilities (WAIS-III Object Assembly subtest). Age and full-scale IQ were partialed out. Among the WS group, greater FA in the right SLF was significantly (and negatively) correlated with visuospatial construction scores $(r=-0.56, p=0.01)$. In contrast, no significant correlations were found between visuospatial construction scores and the FA of left SLF or bilateral ILF. Together, these findings suggest a specific role of right SLF abnormality in visuospatial construction deficits in WS.

\section{Discussion}

These findings strongly support the hypothesis that deficits in visuospatial construction among WS individuals are associated with abnormal white matter integrity along the right SLF. Furthermore, our probabilistic map of the SLF closely resembles SLF II, which extends between the posterior parietal and the posterior lateral prefrontal cortex and is thought to be important for visuospatial attention and processing (Makris et al., 2005). Thus, our findings further establish the association of visuospatial construction abilities with the integrity of SLFII.

We found that the visuospatial deficits in WS were associated with a significant increase in the FA of the right SLF. Our findings suggest that abnormal increases in FA may reliably predict anomalous cognitive function in WS. The cellular mechanisms underlying the increased FA in right SLF among WS remain unknown. However, several factors such as increases in myelination or microscopic deficits of axonal structures or decreases in axonal diameter, packing density, and branching may all contribute to the higher FA that we found (Beaulieu, 2002).

For example, the SLF branches extensively in the frontal, parietal, and temporal lobes in healthy humans. In WS, the volumes of the parietal cortex are substantially reduced compared to controls (after controlling for total brain volume) (Thompson et al., 2005; Eckert et al., 2006a; Meyer-Lindenberg et al., 2006). Also, deletions in WS include the LIM-kinase 1 (LIMK1), a gene that influences neuronal growth, dendritic morphogenesis, and synapse formation (Eckert et al., 2006a). Atypical deletion cases suggest that LIMK1 hemizygosity contributes to WS visuospatial deficits (Eckert et al., 2006a). Thus, one possibility is that dendritic branching limits the number of posterior parietal targets in WS (Eckert et al., 2006a), hence leading to increased FA of the right SLF. This hypothesis would be consistent with findings of reduced brain activation in the parietal region during visuospatial processing (MeyerLindenberg et al., 2004). These interpretations remain to be tested in future studies, because previous studies showed increased FA as potentially reflecting both compensatory mechanisms (Holzapfel et al., 2006) and poor cognitive functioning (Tuch et al., 2005).

Although we attempted to overcome limitations of single analytical approaches by combining three different image-processing techniques, the interpretation of the findings remain limited by the fact that FA is only an indirect marker of white matter microstructural properties and is influenced by a number of factors (Beaulieu, 2002). For example, an increase in FA may be mediated by physiological factors other than those that are related to myelin such as reduced intravoxel fiber crossing or decreased axon diameter. Another parsimonious explanation, which we propose here, is that increased FA in WS is a result of reduced branching.

Although we hypothesized that the ILF would not demonstrate the same degree of abnormal connectivity as SLF, this tract did show significantly increased FA in WS using TBSS (for bilateral IFL) and ROQS (for right ILF) but not with fiber tracking. The ILF extends from the ventral and lateral temporal cortices to the posterior parahippocampal gyrus and is thought to be related to object and face recognition, discrimination, and memory (Schmahmann and Pandya, 2006). The right ILF is an intriguing pathway given WS individuals' known hypersociability and relative proficiency in face recognition (Bellugi et al., 1999), as well as preliminary evidence suggesting atypical function and structure of gray matter regions comprising the ventral "what" pathway (Mobbs et al., 2004; Thompson et al., 2005). Increased ILF findings found in TBSS and ROQS may be caused by an increase in FA in the IFO (which runs in parallel with the ILF in the temporal lobe) rather than ILF, because these techniques are not capable of discriminating between overlapping pathways.

Our study presents the first evidence linking interactions between genetic risk (associated with WS), visuospatial construction, and dorsal white matter fiber anatomy, namely the right SLF. The findings are demonstrated by comparing individuals with WS to two control groups matched for demographics and IQ using three different analytical approaches. The study also shows the first evidence directly associating visuospatial abilities and SLF [note, however, that there is one study that showed FA in a frontoparietal region to correlate positively with visuospatial working memory (Nagy et al., 2004)]. Future studies that investigate in more detail the abnormal white matter regions shown with whole-brain TBSS are warranted. In addition, postmortem studies and investigations of atypical cases with partial 7q11.23 deletions will be of interest in elucidating the specific genes involved in visuospatial construction and its neural substrates.

\section{References}

Basser PJ, Mattiello J, LeBihan D (1994) MR diffusion tensor spectroscopy and imaging. Biophys J 66:259-267.

Beaulieu C (2002) The basis of anisotropic water diffusion in the nervous system-a technical review. NMR Biomed 15:435-455.

Bellugi U, St. George ME, eds (2000) Linking cognitive neuroscience and molecular genetics: new perspectives from Williams syndrome. J Cogn Neurosci 12 [Suppl 1]:1-107.

Bellugi U, Lichtenberger L, Mills D, Galaburda A, Korenberg JR (1999) Bridging cognition, the brain and molecular genetics: evidence from Williams syndrome. Trends Neurosci 22:197-207.

Conturo TE, Lori NF, Cull TS, Akbudak E, Snyder AZ, Shimony JS, McKinstry RC, Burton H, Raichle ME (1999) Tracking neuronal fiber pathways in the living human brain. Proc Natl Acad Sci USA 96:10422-10427.

Derogatis LR (1977) SCL-90: administration, scoring and procedures manual for the revised version and other instruments of the psychopathology rating scale series. Baltimore: John Hopkins University. 
Eckert MA, Hu D, Eliez S, Bellugi U, Galaburda A, Korenberg J, Mills D, Reiss AL (2005) Evidence for superior parietal impairment in Williams syndrome. Neurology 64:152-153.

Eckert MA, Galaburda AM, Mills DL, Bellugi U, Korenberg JR, Reiss AL (2006a) The neurobiology of Williams syndrome: cascading influences of visual system impairment? Cell Mol Life Sci 63:1867-1875.

Eckert MA, Tenforde A, Galaburda AM, Bellugi U, Korenberg JR, Mills D, Reiss AL (2006b) To modulate or not to modulate: differing results in uniquely shaped Williams syndrome brains. NeuroImage 32:1001-1007.

Eckert MA, Galaburda AM, Karchemskiy A, Liang A, Thompson P, Dutton RA, Lee AD, Bellugi U, Korenberg JR, Mills D, Rose FE, Reiss AL (2006c) Anomalous sylvian fissure morphology in Williams syndrome. NeuroImage 33:39-45.

Ewart AK, Morris CA, Atkinson D, Jin W, Sternes K, Spallone P, Stock AD, Leppert M, Keating MT (1993) Hemizygosity at the elastin locus in a developmental disorder, Williams syndrome. Nat Genet 5:11-16.

Galaburda AM, Schmitt JE, Atlas SW, Eliez S, Bellugi U, Reiss AL (2001) Dorsal forebrain anomaly in Williams syndrome. Arch Neurol 58:1865-1869.

Gaser C, Luders E, Thompson PM, Lee AD, Dutton RA, Geaga JA, Hayashi KM, Bellugi U, Galaburda AM, Korenberg JR, Mills DL, Toga AW, Reiss $\mathrm{AL}$ (2006) Increased local gyrification mapped in Williams syndrome. NeuroImage 33:46-54.

Gothelf D, Eliez S, Thompson T, Hinard C, Penniman L, Feinstein C, Kwon H, Jin S, Jo B, Antonarakis SE, Morris MA, Reiss AL (2005) COMT genotype predicts longitudinal cognitive decline and psychosis in 22q11.2 deletion syndrome. Nat Neurosci 8:1500-1502.

Gothelf D, Feinstein C, Thompson T, Gu E, Penniman L, Van Stone E, Kwon H, Eliez S, Reiss AL (2007a) Risk factors for the emergence of psychotic disorders in adolescents with 22q11.2 deletion syndrome. Am J Psychiatry 164:663-669.

Gothelf D, Hoeft F, Hinard C, Hallmayer JF, Van Dover Stoecker J, Antonarakis SE, Morris MA, Reiss AL (2007b) Abnormal cortical activation during response inhibition in 22q11.2 deletion syndrome. Hum Brain Mapp 28:533-542.

Hillier LW, Fulton RS, Fulton LA, Graves TA, Pepin KH, Wagner-McPherson C, Layman D, Maas J, Jaeger S, Walker R, Wylie K, Sekhon M, Becker MC, O'Laughlin MD, Schaller ME, Fewell GA, Delehaunty KD, Miner TL, Nash WE, Cordes M, et al. (2003) The DNA sequence of human chromosome 7. Nature 424:157-164.

Hoeft F, Hernandez A, Parthasarathy S, Watson CL, Hall SS, Reiss AL (2007) Fronto-striatal dysfunction and potential compensatory mechanisms in male adolescents with fragile X syndrome. Hum Brain Mapp 28:543-554.

Holinger DP, Bellugi U, Mills DL, Korenberg JR, Reiss AL, Sherman GF, Galaburda AM (2005) Relative sparing of primary auditory cortex in Williams syndrome. Brain Res 1037:35-42.

Holzapfel M, Barnea-Goraly N, Eckert MA, Kesler SR, Reiss AL (2006) Selective alterations of white matter associated with visuospatial and sensorimotor dysfunction in Turner syndrome. J Neurosci 26:7007-7013.

Jiang H, van Zijl PC, Kim J, Pearlson GD, Mori S (2006) DtiStudio: resource program for diffusion tensor computation and fiber bundle tracking. Comput Methods Programs Biomed 81:106-116.

Kesler SR, Menon V, Reiss AL (2006) Neuro-functional differences associated with arithmetic processing in Turner syndrome. Cereb Cortex 16:849-856.

Korenberg JR, Bellugi U, Salandan LS, Mills DL, Reiss AL (2003) Williams syndrome: a neurogenetic model of human behavior. In: Encyclopedia of the human genome (Cooper D, ed). London: Nature Publishing Group.

Levitin DJ, Menon V, Schmitt JE, Eliez S, White CD, Glover GH, Kadis J, Korenberg JR, Bellugi U, Reiss AL (2003) Neural correlates of auditory perception in Williams syndrome: an fMRI study. NeuroImage 18:74-82.

Makris N, Kennedy DN, McInerney S, Sorensen AG, Wang R, Caviness Jr VS, Pandya DN (2005) Segmentation of subcomponents within the superior longitudinal fascicle in humans: a quantitative, in vivo, DT-MRI study. Cereb Cortex 15:854-869.

Menon V, Leroux J, White CD, Reiss AL (2004) Frontostriatal deficits in fragile X syndrome: relation to FMR1 gene expression. Proc Natl Acad Sci USA 101:3615-3620.

Meyer-Lindenberg A, Mervis CB, Berman KF (2006) Neural mechanisms in
Williams syndrome: a unique window to genetic influences on cognition and behaviour. Nat Rev Neurosci 7:380-393.

Meyer-Lindenberg A, Kohn P, Mervis CB, Kippenhan JS, Olsen RK, Morris CA, Berman KF (2004) Neural basis of genetically determined visuospatial construction deficit in Williams syndrome. Neuron 43:623-631.

Mobbs D, Garrett AS, Menon V, Rose FE, Bellugi U, Reiss AL (2004) Anomalous brain activation during face and gaze processing in Williams syndrome. Neurology 62:2070-2076.

Mobbs D, Eckert MA, Mills D, Korenberg J, Bellugi U, Galaburda AM, Reiss AL (2006) Frontostriatal dysfunction during response inhibition in Williams syndrome. Biol Psychiatry 62:256-261.

Mori S, Crain BJ, Chacko VP, van Zijl PC (1999) Three-dimensional tracking of axonal projections in the brain by magnetic resonance imaging. Ann Neurol 45:265-269.

Mori S, Kaufmann WE, Davatzikos C, Stieltjes B, Amodei L, Fredericksen K, Pearlson GD, Melhem ER, Solaiyappan M, Raymond GV, Moser HW, van Zijl PC (2002) Imaging cortical association tracts in the human brain using diffusion-tensor-based axonal tracking. Magn Reson Med 47:215-223.

Nagy Z, Westerberg H, Klingberg T (2004) Maturation of white matter is associated with the development of cognitive functions during childhood. J Cogn Neurosci 16:1227-1233.

Nichols TE, Holmes AP (2002) Nonparametric permutation tests for functional neuroimaging: a primer with examples. Hum Brain Mapp 15:1-25.

Niogi SN, Mukherjee P, McCandliss BD (2007) Diffusion tensor imaging segmentation of white matter structures using a Reproducible Objective Quantification Scheme (ROQS). NeuroImage 35:166-174.

Committee on Genetics, American Academy of Pediatrics (2001) Health care supervision for children with Williams syndrome. Pediatrics 107:1192-1204.

Reiss AL, Eliez S, Schmitt JE, Straus E, Lai Z, Jones W, Bellugi U (2000) IV. Neuroanatomy of Williams syndrome: a high-resolution MRI study. J Cogn Neurosci 12 Suppl 1:65-73.

Rueckert D, Sonoda LI, Hayes C, Hill DL, Leach MO, Hawkes DJ (1999) Nonrigid registration using free-form deformations: application to breast MR images. IEEE Trans Med Imaging 18:712-721.

Schmahmann JD, Pandya DN (2006) Fiber pathways of the brain. New York: Oxford UP.

Schmitt JE, Eliez S, Bellugi U, Reiss AL (2001a) Analysis of cerebral shape in Williams syndrome. Arch Neurol 58:283-287.

Schmitt JE, Eliez S, Warsofsky IS, Bellugi U, Reiss AL (2001b) Enlarged cerebellar vermis in Williams syndrome. J Psychiatr Res 35:225-229.

Schmitt JE, Eliez S, Warsofsky IS, Bellugi U, Reiss AL (2001c) Corpus callosum morphology of Williams syndrome: relation to genetics and behavior. Dev Med Child Neurol 43:155-159.

Schmitt JE, Watts K, Eliez S, Bellugi U, Galaburda AM, Reiss AL (2002) Increased gyrification in Williams syndrome: evidence using 3D MRI methods. Dev Med Child Neurol 44:292-295.

Smith SM, Jenkinson M, Woolrich MW, Beckmann CF, Behrens TE, Johansen-Berg H, Bannister PR, De Luca M, Drobnjak I, Flitney DE, Niazy RK, Saunders J, Vickers J, Zhang Y, De Stefano N, Brady JM, Matthews PM (2004) Advances in functional and structural MR image analysis and implementation as FSL. NeuroImage [Suppl 1] 23:S208-S219.

Smith SM, Jenkinson M, Johansen-Berg H, Rueckert D, Nichols TE, Mackay CE, Watkins KE, Ciccarelli O, Cader MZ, Matthews PM, Behrens TE (2006) Tract-based spatial statistics: voxelwise analysis of multi-subject diffusion data. NeuroImage 31:1487-1505.

Thompson PM, Lee AD, Dutton RA, Geaga JA, Hayashi KM, Eckert MA, Bellugi U, Galaburda AM, Korenberg JR, Mills DL, Toga AW, Reiss AL (2005) Abnormal cortical complexity and thickness profiles mapped in Williams syndrome. J Neurosci 25:4146-4158.

Tuch DS, Salat DH, Wisco JJ, Zaleta AK, Hevelone ND, Rosas HD (2005) Choice reaction time performance correlates with diffusion anisotropy in white matter pathways supporting visuospatial attention. Proc Natl Acad Sci USA 102:12212-12217.

Wakana S, Jiang H, Nagae-Poetscher LM, van Zijl PC, Mori S (2004) Fiber tract-based atlas of human white matter anatomy. Radiology 230:77-87.

Woods RP, Grafton ST, Holmes CJ, Cherry SR, Mazziotta JC (1998) Automated image registration: I. General methods and intrasubject, intramodality validation. J Comput Assist Tomogr 22:139-152. 